\title{
Incontinência urinária no idoso ${ }^{1}$
}

\author{
Rodolfo Borges dos Reis ${ }^{2}$, Adauto José Cologna ${ }^{3}$, Antonio Carlos Pereira Martins ${ }^{4}$ \\ Edson Luis Paschoalin ${ }^{5}$, Silvio Tucci $\mathbf{J r}^{3}$, Haylton Jorge Suaid ${ }^{3}$
}

\begin{abstract}
Reis RB, Cologna AJ, Martins ACP, Tucci Jr S, Suaid HJ. Incontinência urinária no idoso. Acta Cir Bras [serial online] 2003 vol 18 suppl 5. Disponível em www.scielo.br/acb

RESUMO - A prevalência da incontinência urinária no idoso varia de 8 a $34 \%$ segundo o critério ou método de avaliação. A principais causas são: alterações teciduais da senilidade que comprometem o trato urinário inferior e o assoalho pélvico, do sistema nervoso central e periférico, alterações hormonais como a menopausa, poliúria noturna, alterações psicológicas, hiperplasia prostática benigna, doenças concomitantes e efeitos colaterais de medicamentos. A incontinência pode ser transitória ou permanente. Além da anamnese cuidadosa para caracterização das perdas urinárias, a busca de causas associadas ou concomitantes e o diário miccional, recorre-se com freqüência a exames especializados como a urodinâmica. O diagnóstico preciso é importante para o manejo adequado que pode requerer apenas medidas conservadoras baseadas em orientações e mudanças de hábitos, como o uso de medicamentos, ou então métodos invasivos que incluem procedimentos cirúrgicos específicos.
\end{abstract}

DESCRITORES: Incontinência urinária. Idoso. Noctúria. Urgência.

\section{INTRODUÇÃO}

A incontinência urinária pode ser definida de várias formas. Entretanto, para que possamos comparar resultados de diversos trabalhos científicos e realizar estudos populacionais confiáveis, é necessário uniformizar conceitos e definições. A Sociedade Internacional de Incontinência define incontinência urinária como a condição na qual a perda involuntária de urina é um problema social ou higiênico e é objetivamente demonstrada. A incontinência urinária é muitas vezes erroneamente interpretada como parte natural do envelhecimento. Alterações que comprometem o convívio social como vergonha, depressão e isolamento, freqüentemente fazem parte do quadro clínico, causando grande transtorno aos pacientes e familiares ${ }^{1}$.

Estudos revelam que a prevalência da incontinência urinária no idoso varia de 8 a $34 \% \%^{2,3}$. Essa variação da prevalência pode ser parcialmente explicada pelos diferentes tipos de questionários aplicados, pelas amostras populacionais distintas, pela falta de uniformização nas definições, pela ausência de seguimento a longo prazo das populações estudadas e pelo desconhecimento da história natural da incontinência urinária.

Nos Estados Unidos, a população com 65 anos de idade ou mais cresceu $22 \%$ nos anos 80 . Nos anos 90 , o censo realizado revelou a presença de 31,1 milhões de pessoas com mais de 65 anos e as projeções indicam que no ano de 2050, $21 \%$ da população, aproximadamente 87,4 milhões de pessoas estarão com 65 anos ou mais ${ }^{4}$. O avanço da medicina, aliado à melhora das condições de vida, são por esse aumento da expectativa de vida. Os gastos relacionados à incontinência urinária, nos Estados Unidos, são estimados em 10 bilhões de dólares ao ano ${ }^{5}$. Com o envelhecimento da população, os gastos deverão aumentar. Precisamos estar atentos para a nova realidade e preparados para melhor entender e tratar as enfermidades da terceira idade.

\section{O IMPACTO DA IDADE NA INCONTINÊNCIA URINÁRIA}

Devemos lembrar que a incontinência urinária é um estado anormal e que se realizarmos uma abordagem adequada, é na maioria dos casos resolvida ou minorada. Em qualquer faixa etária, a continência urinária não depende somente da integridade do trato urinário inferior. Alterações da motivação, da destreza manual, da mobilidade, da lucidez e a existência de doenças associadas (diabetes mellitus e insuficiência cardíaca, entre outras) estão entre os fatores que podem ser responsáveis pela incontinência urinária, sem que haja comprometimento significativo do trato urinário inferior. Embora essas alterações sejam raras nos pacientes jovens, são freqüentemente encontradas no idoso e podem agravar ou causar incontinência urinária.
O trato urinário inferior apresenta alterações relacionadas ao envelhecimento, que ocorrem mesmo na ausência de doenças. A força de contração da musculatura detrusora, a capacidade vesical e a habilidade de adiar a micção aparentemente diminuem, no homem e na mulher. Contrações involuntárias da musculatura vesical e o volume residual pós-miccional aumentam com a idade em ambos os sexos. Entretanto, a pressão máxima de fechamento uretral, o comprimento uretral e as células da musculatura estriada do esfíncter alteram-se predominantemente nas mulheres ${ }^{6,7}$.

Além das alterações decorrentes da senilidade dos tecidos, doenças próprias do indivíduo idoso também contribuem para o desenvolvimento de incontinência urinária. A hiperplasia prostática benigna, que está presente em aproximadamente $50 \%$ dos homens aos 50 anos de idade, em metade dos quais causa obstrução ao fluxo urinário e acarreta alterações significativas do trato urinário inferior ${ }^{8}$, como a instabilidade do músculo detrusor. A presença de instabilidade detrusora em muitos indivíduos idosos continentes sugere que a relação entre urge-incontinência e instabilidade detrusora seja mais fraca em pessoas idosas do que em jovens. Uma possível explicação para este fato é que, nos idosos, a característica das contrações involuntárias, de menor amplitude, necessitem de alterações estruturais e funcionais do esfíncter uretral para que ocorra a urgeincontinência. Fatores como o trofismo vaginal

1. Revisão feita no Hospital das Clínicas de Ribeirão Preto - USP

2. Médico Assistente da Divisão de Urologia da FMRP-USP; Responsável pelo Setor de Neurourologia e Incontinência Urinária do Urocenter Ribeirão Preto-SP; Responsável pelo Setor de Neurourologia e Incontinência Urinária do Hospital da Mulher e Maternidade Sinhá Junqueira Ribeirão Preto-SP

3. Prof. Doutor da Divisão de Urologia, Departamento de Cirurgia da FMRP-USP

4. Prof. Titular e Chefe da Disciplina de Urologia, Departamento de Cirurgia - FMRP-USP

5. Pósgraduando do Departamento de Cirurgia - FMRP-USP 
e a presença de distopias também influenciam a presença da urge-incontinência.

Uma das alterações mais freqüentes do hábito urinário no idoso é a noctúria. Alterações hormonais decorrentes do envelhecimento, como o aumento na secreção de vasopressina do hormônio natriurético, podem resultar na eliminação preponderante dos líquidos ingeridos (durante o dia) no período noturno, mesmo na ausência de insuficiência venosa, insuficiência cardíaca, doenças renais ou obstrução prostática. Essas mudanças, associadas à ocorrência de distúrbios do sono fazem com que pessoas com mais de 65 anos apresentem 1 a 2 episódios de noctúria mesmo na ausência de qualquer enfermidade ${ }^{9,10}$. Como essas alterações também são encontradas em indivíduos idosos continentes, não podemos apontar nenhuma delas como causa da incontinência urinária, mas fatores predisponentes que, associados à maior probabilidade dos indivíduos idosos apresentarem alterações psicológicas, efeitos colaterais a medicações e doenças concomitantes, explicam a maior susceptibilidade à incontinência urinária A identificação de fatores que predispõem à incontinência urinária não localizados no trato urinário inferior é de fundamental importância para que possamos abordar a incontinência urinária de maneira adequada.

\section{CLASSIFICAÇÃO DA INCONTINÊNCIA URINÁRIA NO IDOSO}

Para fins didáticos, adotaremos a seguinte subdivisão:

1. Noctúria

2. Incontinência urinária transitória

3. Incontinência urinária persistente

\section{Noctúria}

Levantar-se mais de uma vez por noite para urinar é uma queixa comum que incomoda muitas pessoas de todas as faixas etárias, porém, é mais freqüente dos 50 anos em diante.

\section{Definição}

Várias definições têm sido propostas:

1. é a necessidade de levantar-se da cama mais de uma vez, com o intuito de urinar, após ter ido deitar com a expectativa de só se levantar pela manhã (definição mais aceita);

2. produção de urina, no período noturno, maior que $0,9 \mathrm{ml} / \mathrm{min}^{10}$;

3. produção de urina noturna superior a $33 \%$ da produção diurna (10).

\section{Prevalência}

A prevalência de noctúria na população adulta é de cerca de $10 \%$ quando considerados os indivíduos acima de 20 anos, aumentando para $16 \%$ a partir da quarta década, 26 a $66 \%$ a partir da quinta década e chegando a $55 \%$ nos homens com mais de 70 anos de idade. Apesar da alta prevalência em indivíduos idosos, $63 \%$ dos homens e $59 \%$ das mulheres não procuram auxílio médico $^{11,12}$

\section{Avaliação clínica}

A noctúria, por si, não sugere nenhum diagnóstico específico. A história clínica e o diário miccional do paciente são fundamentais para direcionar a investigação e o tratamento. As principais causas de noctúria são a diminuição da capacidade vesical e/ou o aumento na produção de urina no período noturno. A noctúria pode também estar associada a outros sintomas do trato urinário inferior, a distúrbios do sono ou relacionar-se ao tempo em que o paciente permanece na cama ${ }^{13}$.

As principais causas de aumento da freqüência urinária noturna devido à diminuição da capacidade vesical usual são: diminuição da complacência vesical, hiperatividade detrusora e envelhecimento. As principais causas de poliúria são: diabetes insipidus ou mellitus - produzem mais urina nos períodos diurno e noturno -, insuficiência cardíaca congestiva, hipoalbuminemia, insuficiência venosa e doenças renais. A ingestão noturna de grande quantidade de líquido, cafeína e/ou álcool, assim como o uso de diuréticos, dependendo do horário em que são administrados, merecem destaque na história clínica. $\mathrm{O}$ aumento da produção de vasopressina, que é observado em algumas pessoas com mais de 65 anos de idade, pode também aumentar a produção de urina noturna.

\section{Tratamento}

\section{A - Conservador}

O primeiro passo do tratamento conservador consiste em listar as medicações que o paciente faz uso, na tentativa de detectar alguma que contribua para a noctúria. Nesse caso devemos tentar substituí-la ou alterar a posologia. Nos pacientes com edema periférico e insuficiência cardíaca congestiva, os líquidos acumulam-se nos membros inferiores e são reabsorvidos à noite. Devemos promover a redistribuirão do volume durante o dia através do uso de meias elásticas e da elevação dos membros inferiores no final da manhã e no final da tarde. Dessa forma, o líquido será reabsorvido de maneira uniforme durante o dia, diminuindo a produção de urina no período noturno. A ingestão hídrica deverá ser monitorizada, não permitindo ingestão hídrica 3 horas antes do paciente se deitar.

\section{B - Medicamentoso}

O emprego da desmopressina, de diuréticos, de alfa-bloqueadores e a terapia de reposição hormonal nas mulheres são as opções farmacológicas mais utilizadas.

A alteração do ritmo de liberação da desmopressina verificada em pessoas idosas e a sensibilidade dos túbulos renais a esse hormônio, levounos ao emprego de formas sintéticas do mesmo (DDAVP), visando reduzir a produção de urina e a freqüência urinária noturnas. Os resultados obtidos até hoje nos permitem concluir que o emprego do DDVAP não deve ser rotineiro nos pacientes idosos portadores de noctúria. Embora existam indícios de melhora da sintomatologia, os efeitos colaterais de retenção hídrica e hiponatremia, observados com o emprego da apresentação em forma de "spray" nasal, podem ser perigosos em pacientes idosos portadores de outras doenças. Logo, é recomendado o uso do DDVAP em sua apresentação oral nos pacientes idosos que estejam em boas condições de saúde ${ }^{14,15,16}$

Os diuréticos são boa opção terapêutica, principalmente para aqueles pacientes que apresentam edema periférico ${ }^{17}$. Os diuréticos de alça podem ser usados a qualquer hora do dia, desde que sejam monitorizados os efeitos colaterais de hipotensão postural e distúrbios hidroeletrolíticos.

$\mathrm{O}$ uso de alfa-bloqueadores deve ser reservado para pacientes do sexo masculino, nos quais acreditamos ser o componente prostático a causa mais importante do quadro de noctúria. Devemos advertir os pacientes dos efeitos hipotensores dessa medicação, que ocorrem em $18 \%$ dos pacientes hipertensos.

A terapêutica de reposição hormonal baseia-se na existência de tecidos sensíveis ao estrógeno no assoalho pélvico, na bexiga e na uretra feminina. Sabemos que as mulheres menopausadas apresentam atrofia desses tecidos, com conseqüentes alterações estruturais e anatômicas. Apesar de definido o papel da reposição estrogênica associada à fisioterapia do assoalho pélvico nas pacientes portadoras de incontinência urinária, os resultados até agora obtidos para o tratamento da noctúria são conflitantes, sendo necessários mais estudos para a definição da eficácia desse tratamento ${ }^{18,19}$.

\section{Incontinência Urinária Transitória}

Muitas causas de incontinência urinária em idosos têm origem em locais fora do trato urinário inferior. O risco de incontinência urinária transitória aumenta se ocorrerem, somadas às mudanças fisiológicas do trato urinário inferior, alterações patológicas como infeções, ingestão hídrica excessiva, constipação intestinal crônica, depressão e dificuldade para locomoção.

\section{Definição}

A incontinência urinária transitória é caracterizada pela perda involuntária de urina, precipitada por insulto psicológico, medicamentoso ou orgânico, que cessa ou melhora após o controle do fator desencadeante.

\section{Prevalência}

Apesar da escassez de dados, acredita-se que a incontinência urinária transitória seja responsável por $35 \%$ dos casos de incontinência urinária em asilos de idosos, podendo corresponder a $50 \%$ dos casos de incontinência em pacientes idosos hospitalizados nos Estados Unidos ${ }^{7,20}$.

\section{Avaliação clínica}

A investigação da incontinência urinária transitória baseia-se na avaliação crítica da anamnese e do diário miccional. Pacientes em retenção urinária que apresentam perda de urina por transbordamento (incontinência paradoxal) podem ter como fator desencadeante o emprego de medicações anticolinérgicas na presença de obstrução infravesical ou hipocontractilidade do músculo detrusor. Já o aparecimento ou o agravamento 
da urge-incontinência podem decorrer do uso de diuréticos de alça ou da ingestão excessiva de líquidos por pacientes já portadores de hiperatividade vesical

\section{Causas}

As principais causas de incontinência urinária transitória são:

A - Constipação intestinal - fecaloma

B - Medicamentos

C - Infecção

D - Vaginite atrófica

E - Distúrbios psicológicos

F - Dificuldade de locomoção

G - Ingestão de líquidos em excesso

\section{A - Constipação intestinal}

A presença de fezes impactadas na ampola retal pode ser responsável por até $10 \%$ dos quadros de incontinência urinária atendidos em clínicas geriátricas ${ }^{7}$. A presença de incontinência urinária e fecal, associadas com o achado de fezes impactadas no reto, é comum nos pacientes portadores de incontinência urinária desencadeada por constipação intestinal. A normalização do hábito intestinal e o esvaziamento do fecaloma muitas vezes melhora os sintomas de incontinência urinária nos pacientes idosos.

\section{B - Medicamentos}

Os sedativos e hipnóticos de longa duração, como o diazepam, podem alterar a percepção dos pacientes idosos, levando a episódios de incontinência.

Os diuréticos de alça, como a furosemida, podem aumentar a produção de urina, funcionando como fator predisponente para perdas urinárias.

Quadro 1: Principais drogas e seus efeitos colaterais que podem levar à incontinência urinária.

\begin{tabular}{l|l}
\hline DROGAS & EFEITO \\
\hline Antagonistas alfa-adrenérgicos & Diminuição da resistência uretral \\
\hline Opióides & Constipação intestinal \\
\hline Anticonvulsivantes & Confusão mental / Ataxia \\
\hline Antihipertensivos & Hipotensão arterial - mobilidade diminuída \\
\hline Antiparkinsonianos & Confusão mental / Hipotensão postural \\
\hline Antagonistas H2 & Confusão mental \\
\hline Diuréticos potentes de alça & Aumento da frequiência / Urgência miccional \\
\hline Sedativos / Hipnóticos & Sedação excessiva \\
\hline Anestésicos, raquianestesia, peridural & Paralisia detrusora \\
\hline Antagonistas dos canais de cálcio & Constipação intestinal / retenção urinária \\
\hline Relaxantes musculares & Constipação intestinal / retenção urinária \\
\hline Inibidores da enzima conversora & Tosse - Incontinência urinária de estresse \\
\hline Álcool / Cafeína & Poliúria - aumento da freqüência e urgência miccional \\
\hline
\end{tabular}

\section{D - Vaginite atrófica}

A atrofia do epitélio vaginal, secundária à diminuição dos níveis estrogênicos decorrente da menopausa, é responsável por sintomas do trato urinário inferior nas mulheres. Aproximadamente $80 \%$ das mulheres idosas atendidas em clínicas de incontinência urinária apresentam epitélio
As drogas bloqueadoras dos receptores alfapresentes no colo vesical, ocasionando perdas apresentam como fatores predisponentes a diminuição do comprimento da uretra e da pressão de fechamento uretral.

Os bloqueadores de canal de cálcio, comumente utilizados no controle da hipertensão arterial, levam ao relaxamento da musculatura lisa, ocasionando o aumento do volume residual pósmiccional, podendo gerar incontinência de estresse ou de transbordamento devido à retenção urinária.

Outra classe de droga utilizada no tratamento da hipertensão arterial, que pode levar à incontinência urinária, é a dos inibidores da enzima conversora da angiotensina. Tais drogas podem causar tosse como efeito colateral, precipitando as perdas urinárias de estresse.

Drogas como tranqüilizantes, antidepressivos, antiparkinsonianos, antiespasmódicos e opiáceos podem apresentar efeitos colaterais anticolinérgicos, diminuindo a contractilidade do músculo detrusor e causando retenção urinária, que pode precipitar o aparecimento de incontinência de estresse ou de transbordamento.

O Quadro 1 lista as principais drogas e seus efeitos colaterais que podem levar à incontinência urinária.

\section{C - Infecção}

As infeções do trato urinário podem levar à incontinência urinária, assim como infeções sistêmicas podem levar à confusão mental, ocasionando perdas involuntárias de urina. adrenérgicos podem bloquear os receptores por estresse nas mulheres idosas ${ }^{21}$, já que estas

vaginal fino, friabilidade da muscosa e erosões vaginais. A incontinência urinária relacionada à vaginite atrófica geralmente é acompanhada de urgência miccional e sensação de dor ao urinar. A vaginite atrófica responde muito bem a baixas doses de estrógeno tópico. Esse tratamento também leva à melhora da dispaurenia e à redução dos episódios de cistite senil ${ }^{13}$.

\section{E - Distúrbios Psicológicos}

A depressão e a ansiedade podem levar à disfunção miccional e à incontinência urinária. Os estados de delírio, ao contrário da demência, levam o paciente à confusão mental por períodos que variam de horas a dias. O delírio pode ser causado por medicações ou injúrias agudas ao organismo. Muitas doenças podem se apresentar de maneira atípica nos idosos. Se o paciente entrar em estado de confusão mental, muitas vezes a incontinência urinária é a primeira alteração a ser notada ${ }^{22}$.

\section{F - Dificuldade de Locomoção}

A dificuldade de locomoção é um obstáculo que dificulta a chegada do paciente ao banheiro. Se o indivíduo está acamado por longo período, pode ocorrer hipotensão postural e pós prandial que impedem que o paciente chegue ao banheiro quando necessita urinar. Dependendo da doença de base há associação com alterações da sensibilidade, que podem levar à incontinência urinária Nos pacientes com hiperatividade vesical, a dificuldade de locomoção pode gerar urgeincontinência.

\section{G - Ingestão de líquidos em excesso}

A ingestão excessiva de líquidos pode causar incontinência urinária pelo aumento da produção de urina, principalmente em pacientes acamados, desmotivados ou com distúrbios comportamentais. A associação dessas situações com a hiperatividade vesical aumenta a ocorrência de incontinência urinária. $\mathrm{O}$ diário miccional pode nos fornecer importantes pistas quanto à quantidade de líquido ingerido, a hora da ingestão e a relação com as perdas urinárias. Muitos pacientes apresentam melhora drástica do quadro de incontinência urinária apenas com a mudança dos hábitos relativos à ingestão hídrica.

\section{Incontinência Urinária Persistente}

\section{Definição}

O termo incontinência urinária persistente deve ser empregado quando a perda involuntária de urina não é causada por nenhuma comorbidade existente, não é decorrente do efeito colateral de alguma droga e persiste por pelo menos 3 meses.

\section{Avaliação clínica}

A abordagem clínica inicial deve ser realizada através da história clínica minuciosa, visando excluir causas medicamentosas e comportamentais, além de detectar doenças sistêmicas que possam ser responsáveis pela perda urinária. O diário miccional é fundamental para quantificarmos as perdas e correlacioná-las com os hábitos diários dos pacientes.

\section{Classificação}

Existem 3 tipos de incontinência urinária persistente:

\section{A - Urge-incontinência}

B - Incontinência urinária relacionada ao esvaziamento vesical inadequado

C - Incontinência urinária de esforço 


\section{A - Urge-incontinência}

O termo bexiga hiperativa refere-se aos sintomas clínicos de polaciúria, urgência miccional, noctúria e urge-incontinência. É uma denominação sindrômica, baseada exclusivamente nas queixas clínicas dos pacientes, não dependendo dos achados urodinâmicos. Nos Estados Unidos, a bexiga hiperativa afeta mais de 17 milhões de pessoas. Na Europa, $17 \%$ da população acima dos 40 anos de idade apresenta essa síndrome. Muitos pacientes referem prejuízo significativo da qualidade de vida.

Ao contrário da bexiga hiperativa, a aplicação de outros termos relacionados à urge-incontinência necessita da confirmação urodinâmica da presença de contrações vesicais involuntárias. Contrações vesicais involuntárias são os principais fatores determinantes de urge-incontinência. $\mathrm{O}$ termo bexiga instável relaciona-se à demonstração objetiva de contrações involuntárias vesicais, que podem ser espontâneas ou ocorrer em resposta a estímulos provocativos nas fases de enchimento e armazenamento, durante a cistometria. As contrações devem ser acompanhadas de vontade de urinar. O termo instabilidade detrusora é reservado para os pacientes que apresentam contrações involuntárias documentadas na avaliação urodinâmica e que não apresentam doença neurológica associada. Na presença de fator neurológico desencadeante, devemos usar o termo hiperreflexia do músculo detrusor. Muitas vezes, nos pacientes idosos, não é possível a distinção entre hiperreflexia e instabilidade detrusora.

Dependendo da intensidade das contrações involuntárias e da integridade dos mecanismos de continência a perda involuntária de urina pode ocorrer, sendo chamada de urge-incontinência.

A urge-incontinência é a causa mais freqüente de incontinência urinária permanente nos pacientes idosos ${ }^{23}$. A hiperatividade detrusora pode ocorrer quando a força de contração da musculatura vesical está preservada ou quando está diminuída. Apesar de as duas situações poderem levar à incontinência, os mecanismos envolvidos são diferentes.

A hiperatividade detrusora associada à hipocontractilidade vesical pode levar à urge-incontinência e ao aparecimento de outros sintomas relacionados à obstrução urinária, à incontinência urinária de esforço ou à incontinência por transbordamento. Ao nos depararmos com pacientes idosos que apresentam resíduo pós-

Quadro 2: Principais anticolinérgicos e seus efeitos colaterais.

\begin{tabular}{l|l}
\hline \multicolumn{1}{c|}{ DROGAS } & \multicolumn{1}{c}{ EFEITOS COLATERAIS } \\
\hline Antipsicóticos & Boca seca / confusão mental \\
\hline Antidepressivos tricíclicos & Constipação intestinal \\
\hline Antiparkinsonianos & Confusão mental \\
\hline Sedativos / antihistamínicos & Fadiga / sonolência \\
\hline Antiarrítmicos - disopiramida & Taquicardia \\
\hline Antiespasmódicos & Inibição da contração detrusora \\
\hline Opióides & Retenção urinária / visão turva / Aumento da pressão ocular \\
\hline
\end{tabular}

miccional elevado, é obrigatório fazermos a avaliação da força de contração detrusora para que possamos diferenciar obstrução infravesical de hipocontractilidade do músculo detrusor. Nessa situação especial, o diagnóstico de certeza é obtido através da avaliação urodinâmica.

A hiperatividade detrusora também está presente nos pacientes portadores de obstrução infravesical. Trinta e dois porcento dos homens com obstrução do trato urinário baixo decorrente de hiperplasia prostática benigna, estenose de uretra ou neoplasia prostática apresentam hiperatividade detrusora. Mulheres podem também apresentar quadros de obstrução associados à hiperatividade. Nesses casos, os principais fatores desencadeastes são os prolapsos genitais ou causas iatrogênicas cirúrgicas.

Fatores irritativos locais como tumores vesicais, litíase urinária, divertículos e infeções do trato urinário podem também levar à hiperativade detrusora, com conseqüente urge-incontinência. Nesses casos, acredita-se que os impulsos nervosos direcionados ao sistema nervoso central, gerados pela hipersensibilidade dos receptores sensitivos do trato urinário, causam desequilíbrio entre os estímulos inibitórios, desencadeando a contração vesical.

O tratamento farmacológico da urge-incontinência é baseado na utilização de drogas anticolinérgicas. Já o tratamento conservador consiste na mudança dos hábitos de ingestão hídrica, na realização de fisioterapia do assoalho pélvico, na eletroestimulação e no "biofeedback".

O Quadro 2 lista os principais agentes anticolinérgicos e seus efeitos colaterais.

\section{B - Incontinência urinária relacionada ao esvaziamento vesical inadequado}

A perda involuntária de urina pode estar associada à incapacidade de esvaziar completamente a bexiga. As principais causas de esvaziamento vesical incompleto são a acontractilidade e a hipocontractilidade da musculatura detrusora assim como a obstrução ao fluxo urinário. $\mathrm{O}$ déficit contráctil da musculatura detrusora e a obstrução ao fluxo urinário podem estar presentes isoladamente ou em conjunto. Em situações extremas, a hiperdistenção vesical faz com que ocorra incontinência urinária por transbordamento, também chamada de incontinência paradoxal.
Nos pacientes idosos do sexo masculino a principal causa de esvaziamento vesical deficiente é a hiperplasia benigna da próstata e, menos freqüentemente, o adenocarcinoma prostático e as estenoses da uretra. A ocorrência de obstrução em mulheres é menos freqüente que nos homens e, decorrem da presença de grandes prolapsos genitais ou de complicações das cirurgias para a correção de incontinência urinária. Em ambos os sexos a constipação intestinal, o efeito colateral de drogas como as anticolinérgicas e algumas neuropatias (principalmente a diabética) são causas freqüentes de incontinência relacionada ao esvaziamento vesical deficiente.

A investigação para o diagnóstico da incontinência urinária relacionada ao esvaziamento vesical inadequado baseia-se no diário miccional, na história clínica e nos achados urodinâmicos. O tratamento é direcionado à causa base, podendo ser simplesmente a interrupção de alguma droga que o paciente esteja utilizando, ou procedimentos cirúrgicos que visem a desobstrução do fluxo urinário.

\section{C - Incontinência urinária de esforço}

A incontinência urinária induzida pelo estresse é uma das causas mais comuns de perda involuntária de urina nos pacientes idosos do sexo feminino $^{24,25}$. A causa mais freqüente de incontinência urinária de esforço é a hipermotilidade uretral decorrente da fraqueza do assoalho pélvico ou conseqüente a procedimentos cirúrgicos. A deficiência esfincteriana intrínseca também é freqüientemente observada e está geralmente associada à desnervação do assoalho pélvico, à radioterapia prévia, à diminuição dos níveis estrogênicos e a procedimentos cirúrgicos. A instabilidade uretral, que consiste no relaxamento esfincteriano na ausência de contração vesical, é uma causa rara de incontinência urinária de esforço e de difícil diagnóstico.

Pacientes idosos do sexo masculino raramente apresentam incontinência urinária de esforço. Quando isso ocorre, a principal causa é o funcionamento inadequado do mecanismo esfincteriano decorrente de radioterapia prévia ou de procedimentos cirúrgicos, principalmente as prostatectomias, quer realizadas para doenças prostáticas benignas ou malignas.

O tratamento da incontinência urinária de esforço depende da causa base, das condições clínicas do paciente e da expectativa em relação aos resultados. Atualmente existem 3 formas de abordagem terapêutica:

\section{- Tratamento farmacológico;}

- Tratamento conservador comportamental e fisioterápico;

\section{- Tratamento cirúrgico}

A forma de tratamento deve ser decidida correlacionando-se os dados da avaliação clínica e dos achados urodinâmicos, quando estes estiverem disponíveis. As modalidades terapêuticas citadas acima não são obrigatoriamente utilizadas individualmente. A combinação de diferentes estratégias muitas vezes proporciona resultados melhores, com menor agressão ao paciente. 


\section{CONCLUSÃO}

A incontinência urinária é erroneamente vista como um processo natural do envelhecimento. As melhorias dos indicadores de saúde, como o aumento da expectativa de vida, assim como o avanço da medicina fazem com que observemos o fenômeno do envelhecimento da população. Cada vez mais devemos estar atentos e aptos a tratar as doenças do idoso, melhorando dessa forma sua qualidade de vida.

\section{REFERÊNCIAS}

1. Wyman JF, Harkins SW, Fantl JA. Psychosocial impact of urinary incontinence in the community dwelling population. J Am Geriatric Soc 1990; 38:282-8.

2. Herzog AR, Fultz NH: Prevalence and incidence in community-dwelling populations. J Am Geriatric Soc 1990; 38: 273-8.

3. Holst K, Wilson PD. The prevalence of female urinary incontinence and reasons for not seeking treatment. N Z Med J 1988; 9:756-61.

4. US Bureau of the Census. Sixty-Five Plus in America. Currente Populations Reports, Special Studies, P23-178. Washington, DC, US Government Printing Office, 1992

5. Legace EA, Hansen W, Hickner JM. Prevalence and severity of urinary incontinence in ambulatory adults: Ann UPRNet study. J Fam Pract 1993; 36:6105 .

6. Diokono AC, Brown MB, Brock BM. Clinical and cystometric characteristics of continente and incontinent noninstitutionalized elderly. J Urol 1988; 140:567-71.

7. Resnick NM. Voiding dysfunction in the elderly. In Yalla SV, McGuire EJ, Elbadawi A, Blaivas JG: Neurourology and
Urodynamics: Principles and Practice. New York, MacMillam Publishing Company, $\mathrm{p}$ 303, 1988

8. Resnik NM, Elbadawi A, Yalla SV. Age and the lower urinary tract: What is normal? Neurourol Urodyn 1997; 12: 322-30.

9. Herzog AR, Diokono AC, Fultz NH. Urinary incontinence: medical and psychosocial aspects. Annu Re Gerontol Geriatr 1989; 9:74-119.

10. Miller M. Nocturnal polyuria in older people: Pathophysiology and clinical implications. J Am Geriatr Soc 2000; 48:1321.

11. Perry S, Shaw C, Assassa P, Dallosso H, Williams K. Ann epidemiological study to establish the prevalence of urinary symptoms and felt need in thr community: The leicestershire MRC incontinence study: J Pub Heal Med 2000; 22:427.

12. Pinnock CB, Marshall VR. Troublesome lower urinary tract symptoms in the community: a prevalence study. Med J Aust 1997; 56:72.

13. Pressman MR, Figueroa WG, KenrickMohamMed J, Greenspon LW, Peterson DD. Noctúria: a rarely, recognized symptom of sleep apnoea and other occult sleep disorder. Arc Int Med 1996; 347:270.

14. Asplund R, Sunberg B, Bengtsson P. Desmopressin for the treatment of nocturnal polyuria in the elderly: a dose titration study. Br J Urol 1993; 72: 38.

15. Chancelor MB, Atan A, Rivas D, Watanabe T, Tai HL, Kumon H. Beneficial effect of intranasal desmopressin for men with benign prostatic hyperplasia and nocturia. Techniques in Urology 1999; 5: 191.

16. Seiler WO, Stahelin HB, Hefti U. Desmopressin reduces night urine volume in geriatric patients: Implications for treatment of the nocturnal incontinence, Clin Ivest 1992; 70: 619.
17. Reynard JM, Cannon A, Yang Q, Abrams P. A novel therapy for nocturnal polyuria: a double blind randomized trail of furosemide against placebo. Br J Urol 1998; 81: 215 .

18. Kok ALM, Burger CW, Van De Weijer PHM. Micturition complaints in postmenopausal women treated with continuously combined hormone replacement therapy: a prospective study. Maturitas 1999; 31: 143.

19. Fantl J, Bump R, Robinson D, McClish D, Wyman J. Efficacy of stogen suplementation in treatment of urinary incontinence. Obs \& Gyn 1996; 88: 745.

20. Brandeis GB, Baumann MM, Hossain M, Morris JN, Resnick NM. The prevalence of potentially remediable urinary incontinence in frail elderly people: A study using the Minimum Data Set. J Amer Geriatr Soc 1997; 45:179.

21. Marshall HJ, Beevers DG. Alfa-adrenoceptor blocking drugs and female urinary incontinence: prevalence and reversibility. Br J Clin Pharmacol 1996; 42:507.

22. Raz R, Stamm WE. A controlled trial of intravaginal estriol in postmenopausal women with recurrent urinary tract infection. N Engl J Med 1997; 329: 753.

23. Diokono AC, Wells TJ, Brink CA. Comparison of self-reported voided volume with cistometric bladder capacity. J Urol 1987; 137:698.

24. Dikono AC, Wells TJ, Brink CA. Urinary incontinence in the elderly women: urodynamic evaluation. J Amer Geriatr Soc 1987; 35: 940.

25. Wells TJ, Brink CA, Dikono AC. Urinary incontinence in elderly women: clinical findings. J Ame Geriatr Soc 1987; $35: 933$.

\begin{abstract}
Reis RB, Cologna AJ, Martins ACP, Tucci Jr S, Suaid HJ. Urinary incontinence in the elderly.. Acta Cir Bras [serial online] 2003 vol 18 suppl 5. Available in www.scielo.br/acb

ABSTRACT - The prevalence of urinary incontinence in the elderly varies from 8 to $34 \%$ according to the criteria or method of investigation. The etiology or main associated factors are: aging tissular degeneration that compromise the lower urinary tract and pelvic floor, changes of peripheric and central nervous system, hormonal alterations such as menopause, nocturnal polyuria, benign prostate hyperplasia, concomitant diseases and side effects of medical drugs. The incontinence may be transitory or permanent. Besides a criterious medical history for a better characterization of the urinary loss, a search for associated or concomitant causes

and the miccional diary, one oftenly may rely on specialized exams such as urodynamics. A specific diagnosis is of utmost value for correct management that may require only conservative measures based on changes of behaviour or counceiling, drugs prescription, or invasive methods including surgical procedures.
\end{abstract}

KEY WORDS: Urinary incontinence. Elderly. Nocturia. Urgency.

\title{
Correspondência
}

Rodolfo Borges Reis

Departamento de Cirurgia e Anatomia - HCFMRP-USP, $9^{\circ}$ Andar

Av. Bandeirantes, 3900, CEP: 14048-900

Ribeirão Preto, SP 